Document downloaded from:

http://hdl.handle.net/10251/112948

This paper must be cited as:

Martorell-Aygues, P.; Marton Lluch, I.; Sánchez Galdón, Al.; Martorell Alsina, SS. (2017). Unavailability model for demand-caused failures of safety components addressing degradation by demand-induced stress, maintenance effectiveness and test efficiency. Reliability Engineering \& System Safety. 168:18-27. doi:10.1016/j.ress.2017.05.044

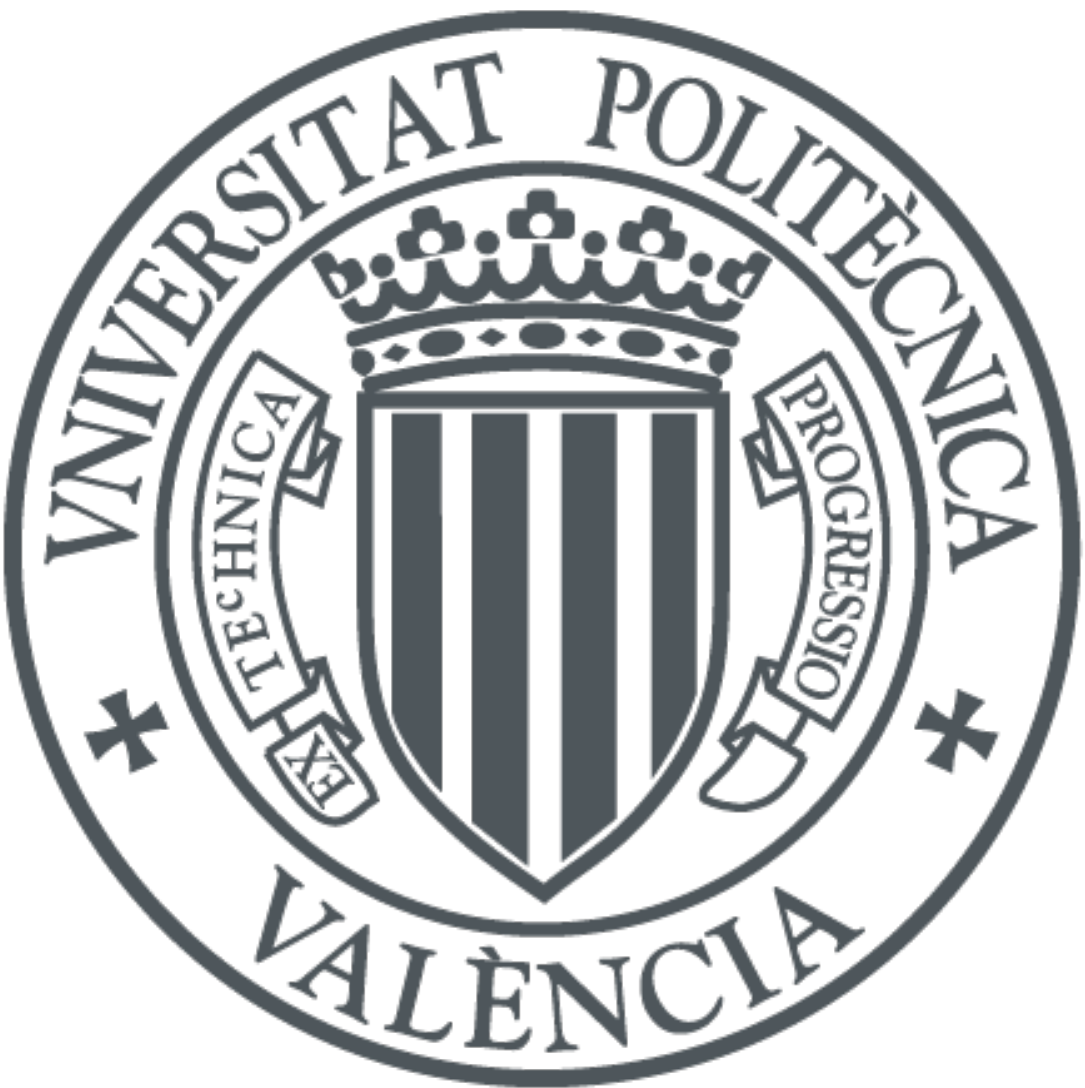

The final publication is available at

https://doi.org/10.1016/j.ress.2017.05.044

Copyright Elsevier

Additional Information 


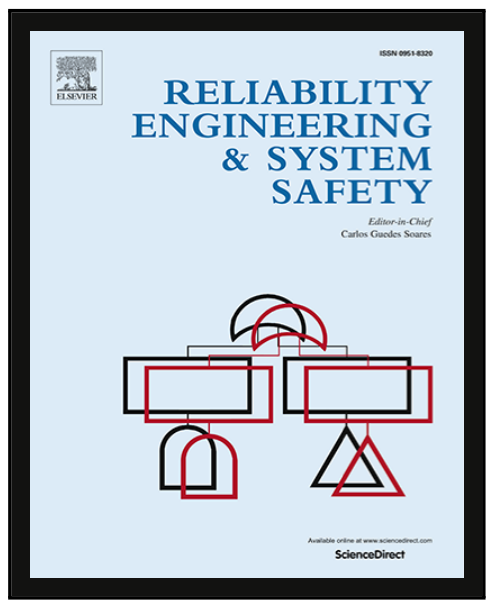

Unavailability model for demand-caused failures of safety components addressing degradation by demand-induced stress, maintenance effectiveness and test efficiency

P. Martorell , I. Martón , A.I. Sánchez , S. Martorell

PII: S0951-8320(16)30920-6

DOI: 10.1016/j.ress.2017.05.044

Reference: $\quad$ RESS 5864

To appear in: $\quad$ Reliability Engineering and System Safety

Received date: $\quad 30$ November 2016

Revised date: $\quad 12$ May 2017

Accepted date: 27 May 2017

Please cite this article as: P. Martorell, I. Martón, A.I. Sánchez, S. Martorell, Unavailability model for demand-caused failures of safety components addressing degradation by demand-induced stress, maintenance effectiveness and test efficiency, Reliability Engineering and System Safety (2017), doi: 10.1016/j.ress.2017.05.044

This is a PDF file of an unedited manuscript that has been accepted for publication. As a service to our customers we are providing this early version of the manuscript. The manuscript will undergo copyediting, typesetting, and review of the resulting proof before it is published in its final form. Please note that during the production process errors may be discovered which could affect the content, and all legal disclaimers that apply to the journal pertain. 


\section{Highlights}

- New model for the demand failure probability of a safety component

- Demand-related stress, maintenance effectiveness and test efficiency addressed explicitly for demand-caused unreliability modelling

- Study of maintenance and test-related parameters

- Unavailability model extension of a component normally in standby

- Positive and negative effects of surveillance and maintenance activities 


\title{
Unavailability model for demand-caused failures of safety components addressing degradation by demand-induced stress, maintenance effectiveness and test efficiency
}

\author{
P. Martorell ${ }^{1,3}$, I.Martón ${ }^{1,3}$, A.I. Sánchez ${ }^{2,3}$, S. Martorell ${ }^{1,3^{*}}$ \\ València, Valencia, Spain \\ València, Valencia, Spain \\ ${ }^{3}$ MEDASEGI group (http://medasegi.webs.upv.es/home/) \\ *Corresponding author: smartore@iqn.upv.es
}

${ }^{1}$ Department of Chemical and Nuclear Engineering. Universitat Politècnica de

${ }^{2}$ Department of Statistics and Operational Research. Universitat Politècnica de

\author{
ABSTRACT
}

The reliability, availability and maintainability (RAM) modelling of safety equipment has long been a topic of major concern. Some RAM models have focussed on explicitly addressing the effect of component degradation and surveillance and maintenance policies, searching for an optimum level of the safety component RAM by adjusting surveillance and maintenance related parameters. As regards the reliability contribution, these components normally have two main types of failure mode that contribute to the probability of failure on demand (PFD): (1) by demand-caused and (2) standby-related failures. The former is normally associated with a demand failure probability, which is affected by the degradation caused by demand-related stress. Surveillance testing therefore not only introduces a positive effect, but also an adverse one, which it compensates by performing maintenance activities to eliminate or reduce the accumulated degradation. This paper proposes a new model for the demand failure probability that explicitly addresses all aspects of the effect of demand-induced stress (mostly test-induced stress), maintenance effectiveness (PAS or PAR model) and test efficiency. A case study is included on an application to a typical motor-operated valve in a nuclear power plant.

Keywords: Unavailability, unreliability, demand induced stress, degradation, imperfect maintenance, test stress, test efficiency, maintenance effectiveness, NPP safety components

*Corresponding author: smartore@iqn.upv.es 


\title{
ACRONYMS AND NOTATION
}

\author{
BAO Bad As Old \\ GAN Good As New \\ MOV Motor Operated Valve \\ NPP Nuclear Power Plant \\ PAR Proportional Age Reduction
}

PAS Proportional Age Set-Back

PFD Probability of Failure on Demand

PSA Probabilistic Safety Analysis

RAM Reliability, Availability, Maintainability

$\alpha \quad$ Linear ageing rate

$t \quad$ Chronological time

$t^{\prime} \quad$ Time elapsed since the last test

$t_{D} \quad$ Chronological time at which the initial component unreliability is doubled

$v \quad$ Time elapsed since the last overhaul point

$T \quad$ Test interval

$\eta \quad$ Test efficiency

M Preventive maintenance interyal

$m \quad$ Preventive maintenance number $m$

$m_{D} \quad$ Preventive maintenance at which the initial component unreliability is doubled

$\mathrm{m}^{*} \quad$ Preventive maintenance at which the component is replaced by a new one

$\varepsilon \quad$ Preventive maintenance effectiveness

L Replacement interval (overhaul maintenance)

$n(t)$ Accumulated number of demands at time $t$

$n_{T D}(t)$ Number of surveillance test performed up to time $t$

$\rho_{0} \quad$ Residual demand failure probability

$p_{1} \quad$ Test degradation factor associated with demand failures

$p_{2} \quad$ Test degradation factor associated with standby failures

$f(t) \quad$ Degradation function associated with demand-related stress

$f_{m}(t)$ Time-dependent evolution of the degradation function of the component over the period between maintenance $m-1$ and $m$ 
$f_{m}^{+} \quad$ Time-dependent degradation function immediately after maintenance $m$

$f_{m}^{-} \quad$ Time-dependent degradation function immediately before maintenance $m$

$\rho(f)$ Time-dependent demand failure probability

$\rho_{m}(f)$ Time-dependent evolution of the demand failure probability over the period between maintenance $m-1$ and $m$

$\rho_{m}^{+} \quad$ Time-dependent demand failure probability immediately after maintenance $m$

$\rho_{m}^{-} \quad$ Time-dependent demand failure probability immediately before maintenance $m$

$\rho_{m} \quad$ Averaged demand failure probability between maintenance activities $m-1$ and $m$

$\rho^{*} \quad$ Averaged demand failure probability over the component useful life, i.e. over the renewal period $L$

$\tau \quad$ Downtime for testing

$\sigma \quad$ Downtime for preventive maintenance

$\mu \quad$ Downtime for corrective maintenance or repair

$\theta \quad$ Downtime for replacement or renewal

$u_{R}(t)$ Time-dependent unreliability of the component

$u_{R, m}(t)$ Time-dependent evolution of the component unreliability over the period between maintenance $m-1$ and $m$

$u_{R, m}^{+} \quad$ Time-dependent component unreliability immediately after maintenance $m$

$u_{R, m}^{-} \quad$ Time-dependent component unreliability immediately before maintenance $m$

$u_{R, m} \quad$ Averaged unreliability over the period between maintenance $m-1$ and $m$

$u_{R} \quad$ Unreliability contribution to the component averaged unavailability over the component useful life, i.e. over the renewal period $L$

$u \quad$ Total averaged unavailability

$u_{T} \quad$ Averaged unavailability contribution due to testing

$u_{M}$ Averaged unavailability contribution due to performing preventive maintenance

$u_{C} \quad$ Averaged unavailability contribution due to performing corrective maintenance

$u_{O}$ Averaged unavailability contribution due replacement or component renewal 


\section{INTRODUCTION}

The safety of nuclear power plants (NPPs) depends on the availability of safety-related components that are normally on standby and only operate in the case of a true demand. The probabilistic risk assessment (PRA) of a NPP normally considers a basic unreliability model for these safety-related components, which usually have two main types of failure modes that contribute to the probability of failure on demand (PFD): (1) by demand-caused and (2) standby-related failures. The former is often associated with a demand failure probability $(\rho)$, and the latter with a standby failure rate $(\lambda)$. Both are generally associated with constant values in a standard PRA, i.e. $\rho_{0}$ and $\lambda_{0}$ respectively, which do not take into account the component degradation due to demand-induced stress and ageing.

However, both failure modes are often affected by degradation such as demand-related stress and ageing, which cause the component to degrade and ultimately to fail. Maintenance and test activities are performed to control degradation and the unreliability and unavailability of such components, although this has both positive and negative effects.

Early studies reported in [1, 2] have provided a well-organized foundation for the positive and adverse effects of testing these components, accounting for both by demand-caused and standby-related failure modes. Kim et al. (1991) [1] proposed a well-organized foundation to account for ageing and the positive and adverse effects of testing the components in modelling demand failure probability and standby failure rate, which represents a more realistic unreliability modelling of safety components. Kim et al. (1994) [2] later proposed a simplification of the earlier unreliability model, which can be formulated as follows:

$$
u_{R}\left(n, t^{\prime}\right)=\rho(n)+\int_{n T}^{n T+t \prime} \lambda(n, u) d u \quad \text { for } \quad t^{\prime} \in[0, T]
$$

being the demand-caused unreliability contribution

$$
\rho(n)=\rho_{0}+\rho_{0} p_{1} n
$$


and the standby-related unreliability contribution:

$$
\lambda(n, u)=\lambda_{0}+\lambda_{0} p_{2} n+\alpha v \quad \text { for } \quad v \in\left[0, \mathrm{nT}+\mathrm{t}^{\prime}\right]
$$

where,

$n=$ number of test performed on the equipment at chronological time $t$

$T=$ test interval

$t^{\prime}=$ time elapsed since the last test

$v=$ time elapsed since the last overhaul point

$\rho_{0}=$ residual demand failure probability

$p_{l}=$ test degradation factor associated with demand failures

$p_{2}=$ test degradation factor associated with standby failures

$\lambda_{0}=$ residual standby time-related failure rate

$\alpha=$ aging factor associated with ageing alone

Eqs.(1) to (3) represent the unreliability model that can estimate the probability of failure on demand (PFD) of a safety component, considering both failure modes on demand, i.e. by demand and standby-related, and at the same time integrates component degradation due to test-induced stress and linear ageing. It also addresses the positive effect of testing, i.e. whether the test is one hundred per cent effective in detecting both demand-caused and standby-related failures.

However, this model does not take into account other important positive and negative effects on the component unreliability, such as: (1) the positive effect of maintenance activities as a function of their effectiveness in managing component degradation due to demand-induced stress and ageing, (2) the negative effect of test inefficiency in detecting failures, (3) demand-induced stress other than that due to testing, e.g. real demands.

As regards the standby-related failure mode, some studies have found that the standby failure rate of a safety component is affected by both demand-induced stress and ageing. Thus, Martorell et al. (1999) [3] provided an age-dependent reliability model associated only with standby-related failures that explicitly takes into account the effect of 
equipment ageing and the positive and negative effects of maintenance activities founded on imperfect maintenance modelling. Martón et al. (2015) [4] recently proposed an approach to modelling the unavailability of safety-related components associated with standby-related failures that explicitly addresses all aspects of the effect of ageing, maintenance effectiveness and test efficiency. These models do not take into account the explicit degradation effects due to demand-induced stress. Other authors have proposed alternative approaches to modelling the effect of ageing and test and maintenance activities [5-8].

As regards the demand-caused failure mode, this probability of a safety component is normally considered to be mainly affected by demand-induced stress, e.g. due to true demands, proof tests and others. The demand-induced stress is therefore modelled with a stochastic degradation jump in Refs. [9-12], without aceounting for test-induced degradation. These studies consider that random shocks occur according to a Non Homogeneous Poisson Process, leading to the immediate failure of the component. Torres Echeverría et al. $(2009 ; 2011)$ [13,14] provided a model to address the effects of test strategies on the probability of failure on demand for safety instrumented systems. And Sung Min Shin et al. (2015) [15] recently proposed an age-dependent model that considers among others, the effect of "test stress" and maintenance effects. In general, previous studies have found that the demand failure probability should be considered a function not only of the number of tests but also of the effectiveness of maintenance activities.

In this context, this paper focuses on the demand-caused failure mode and proposes a new model for the demand failure probability of a safety component based on modelling the standby-related failure mode proposed in Martón et al. (2015) [4], explicitly addressing all aspects of the effect of demand-induced stress, maintenance effectiveness and test efficiency.

The rest of this paper is organized as follows: Section 2 focuses on the development of a demand failure probability model that addresses component degradation as a consequence of demand-induced stress and the positive effect of preventive maintenance. Section 3 describes the unavailability model of a component that is affected only by demand-caused failures, which consists of not only the unreliability 
contribution associated with the previous demand failure probability model but also the component unavailability contributions associated with downtimes for testing and maintenance, therefore considering other negative effects of both test and maintenance activities. Lastly, Section 4 describes an application in a case study involving a motoroperated valve.

\section{DEMAND FAILURE PROBABILITY MODELLING ADDRESSING DEMAND- INDUCED STRESS, MAINTENANCE EFFECTIVENESS AND TEST}

\section{EFFICIENCY}

\subsection{Demand failure probability addressing demand-induced stress}

Considering the contributions formulated in Eq. (2), the demand failure probability of a component normally on standby and ready to perform a safety function on demand depends on the number of demands on the component. This number is often not only associated with performing the planned surveillance or functional tests, but also with those associated with unplanned operational demands and tests performed after a repair of a component failure.

Operational demands are defined as a manual or automatic initiation of a component that was not part of a pre-planned evolution, such as testing. The frequency of unplanned demands can be estimated from the operational experience of the plant as the number of unplanned demands divided by operating time (in years). For example, NPP high pressure coolant injection and reactor core isolation cooling turbine-driven pumps have historically experienced demands in the order of about once every 2.5 reactoryears

Demands associated with functional and surveillance tests are usually laid down in nuclear power plant Technical Specifications, which require either a full demand or a simulation of the automatic start of the safety components. Thus, for example, the typical frequency of a functional test can be 18 months, i.e. equivalent to a refuelling cycle (0.66 per year), while typical values of surveillance tests are one per week (52 per 
year), once per month (12 per year), quarterly (4 per year), etc. depending on the importance of the safety function of the component.

Test-induced stress is therefore not the only relevant contribution to demand-induced stress, especially for components with a low test frequency. Nevertheless, the most important components often undergo more surveillance tests and as a consequence testinduced stress will probably be the most significant contribution to their demandinduced stress. Accordingly, the time-dependent demand failure probability given by Eq. (2) could be reformulated in terms of a time-dependent degradation function, $f(t)$, as follows:

$$
\rho(t)=\rho_{0}+\rho_{0} * f(t)
$$

In current PRA modelling, Eq. (4) can be simplified as follows: $\rho(t)=\rho_{0}$, due to not explicitly accounting for the degradation associated with demand-induced stress. Nevertheless, these effects are limited implicitly by adopting a living PRA or at least updating the standard PRA regularly, which is mandatory by current regulation in many countries. Assuming that the degradation factor is the same for all types of demands and is equal to $p_{1}, f(t)$ can be formulated as follows:

$$
f(t)=p_{1} * n(t)
$$

Where $n(t)$ should include the number of surveillance test, number of functional test, number of operational demands, number of test after preventive maintenance and number of test after corrective maintenance performed up to time $t$.

When only test-induced stress is considered, the demand failure probability is represented by Eq. (4), while Eq. (5) simplifies for $n(t)=\left\lceil\frac{t}{T}\right\rceil$, where $T$ represents the test interval and $\lceil x\rceil$ the floor function that gives the largest integer less than or equal to 
$x$. This simplification is adopted in the following sections without loss of generality and for the sake of simplicity in the introduction and formulation of the following positive and negative effects of performing maintenance and tests.

\subsection{Addressing maintenance effectiveness}

In order to introduce the positive effect of preventive maintenance on component demand-induced stress degradation, two imperfect maintenance models are considered in this paper: Proportional Age Reduction (PAR) and Proportional Age Setback (PAS) models $[3,16]$.

\subsubsection{PAS model}

In the PAS approach, each maintenance activity is assumed to proportionally reduce, by a factor $\varepsilon$, the degradation of the component immediately before it enters maintenance, where $\varepsilon$ represents the maintenance effectiveness that ranges in the interval $[0,1]$.

Time-dependent demand failure probability, $\rho_{m}(t)$

Thus, considering that the degradation function $f($.$) depends only on the number of$ surveillance tests, as given by Eq. (7), the degradation function can be formulated for the time immediately before and after the first maintenance activity, named $f_{1}^{-}$and $f_{1}^{+}$respectively, as follows:

$$
\begin{aligned}
& f_{1}^{-}=p_{1} \cdot\left\lceil\frac{t_{1}}{T}\right\rceil \\
& f_{1}^{+}=p_{1} \cdot(1-\varepsilon) \cdot\left\lceil\frac{t_{1}}{T}\right\rceil
\end{aligned}
$$

being $\varepsilon$ the maintenance effectiveness, $T$ the surveillance test interval and $\lceil x\rceil$ the floor function that gives the largest integer less than or equal to $x$. Thus, in Eqs. (6) and (7) 
the term $\left\lceil\frac{t_{1}}{T}\right\rceil$ represents the number of tests already performed on the component up to the first maintenance activity, arriving at chronological time $t=t_{1}$.

Next, the degradation function can be formulated for the time immediately before and after the second maintenance activity, arriving at chronological time $t_{2}$ as follows:

$$
\begin{aligned}
& \mathrm{f}_{2}^{-}=p_{1} \cdot(1-\varepsilon) \cdot\left\lceil\frac{\mathrm{t}_{1}}{T}\right\rceil+p_{1} \cdot\left\lceil\frac{\left(\mathrm{t}_{2}-\mathrm{t}_{1}\right)}{T}\right\rceil \\
& \mathrm{f}_{2}^{+}=p_{1} \cdot(1-\varepsilon)\left[(1-\varepsilon) \cdot\left\lceil\frac{\mathrm{t}_{1}}{T}\right\rceil+\left\lceil\frac{\left(\mathrm{t}_{2}-\mathrm{t}_{1}\right)}{T}\right\rceil\right]
\end{aligned}
$$

In general, the degradation function after maintenance number $m$ can be formulated by:

$$
\begin{aligned}
& \mathrm{f}_{\mathrm{m}}^{+}=p_{1} \cdot\left\{(1-\varepsilon)^{\mathrm{m}} \cdot\left\lceil\frac{\mathrm{t}_{1}}{T}\right\rceil+(1-\varepsilon)^{\mathrm{m}-1} \cdot\left\lceil\frac{\left(\mathrm{t}_{2}-\mathrm{t}_{1}\right)}{T}\right\rceil+\cdots+(1-\varepsilon) \cdot\left\lceil\frac{\left(\mathrm{t}_{\mathrm{m}}-\mathrm{t}_{\mathrm{m}-1}\right)}{T}\right\rceil\right\}= \\
& p_{1} \cdot\left\{\sum_{\mathrm{k}=1}^{\mathrm{m}}(1-\varepsilon)^{\mathrm{k}} \cdot\left\lceil\frac{\mathrm{t}_{\mathrm{m}-\mathrm{k}+1}-\mathrm{t}_{\mathrm{m}-\mathrm{k}}}{T}\right\rceil\right\}
\end{aligned}
$$

Now, assuming preventive maintenance activities are performed on a regular basis with a constant maintenance interval given by $M$, Eq. (10) can be simplified as follows:

$$
\begin{gathered}
\mathrm{f}_{\mathrm{m}}^{+}=p_{1} \cdot \frac{M}{T} \cdot \sum_{\mathrm{k}=1}^{\mathrm{m}}(1-\varepsilon)^{\mathrm{k}}=p_{1} \cdot \frac{M}{T} \cdot \sum_{\mathrm{k}=0}^{\mathrm{m}-1}(1-\varepsilon)^{\mathrm{k}+1} \\
=p_{1} \cdot \frac{M}{T} \cdot(1-\varepsilon) \sum_{\mathrm{k}=0}^{\mathrm{m}-1}(1-\varepsilon)^{\mathrm{k}}
\end{gathered}
$$

The last term on the right hand side of Eq. (11) corresponds to the summation of a geometric series in which 1 is the first term and $(1-\varepsilon)$ is the common ratio. Then, Eq. (11) can be reformulated as follows:

$$
\mathrm{f}_{\mathrm{m}}^{+}=p_{1} \cdot \frac{M}{T} \cdot(1-\varepsilon) \cdot \frac{1-(1-\varepsilon)^{m}}{1-(1-\varepsilon)}=p_{1} \cdot \frac{M}{T} \cdot \frac{(1-\varepsilon)}{\varepsilon} \cdot\left\{1-(1-\varepsilon)^{m}\right\}
$$


and similarly, it is possible to derive the following generalization for the degradation function before maintenance number $m$ :

$$
\mathrm{f}_{\mathrm{m}}^{-}=p_{1} \cdot \frac{M}{T} \cdot \frac{1-(1-\varepsilon)^{m}}{\varepsilon}
$$

It is quite simple to demonstrate that Eqs. (12) and (13) represent monotonically increasing functions of $m$, which have an asymptotic behaviour given respectively by:

$$
\begin{aligned}
& \mathrm{f}_{\mathrm{a}}^{+}=p_{1} \cdot \frac{M}{T} \cdot \frac{(1-\varepsilon)}{\varepsilon} \\
& \mathrm{f}_{\mathrm{a}}^{-}=p_{1} \cdot \frac{M}{T} \cdot \frac{1}{\varepsilon}
\end{aligned}
$$

Now, it is possible to extend the time-dependent degradation function given by Eq. (5) to account for the effect of imperfect maintenance following a PAS model. Thus, in the period number $m$, i.e. between maintenance $m-1$ and $m$, the evolution of the degradation function of the component can be represented by:

$$
f_{m}(t)=f_{m-1}^{+}+p_{1} \cdot\left[\frac{t-t_{m-1}}{T}\right]
$$

Using Eq. (4), a time-dependent demand failure probability model that addresses the demand-induced stress and the effect of $m-1$ maintenance activities can be formulated for the period $m$ as follows:

$$
\rho_{m}(t)=\rho_{0}+\rho_{0} \cdot f_{m}(t)
$$

Eq. (17) can be particularized for $t=t_{m}$ immediately after performing maintenance number $m$ making use of Eqs. (16) and (12), which yields the formulation of the timedependent demand failure probability immediately after maintenance $m$ :

$$
\rho_{\mathrm{m}}^{+}=\rho_{m}\left(t=t_{m}\right)=\rho_{0}+\rho_{0} \cdot p_{1} \cdot \frac{M}{T} \cdot \frac{(1-\varepsilon)}{\varepsilon} \cdot\left\{1-(1-\varepsilon)^{m}\right\}
$$

Eq. (18) can be reformulated as follows: 


$$
\rho_{\mathrm{m}}^{+}=\rho_{0}+\Delta \rho_{m}^{+}
$$

Where $\Delta \rho_{m}^{+}$represents the increase in the time-dependent demand failure probability of the component due to demand-caused degradation accumulated up to having performed maintenance activity number $m$, which is given by:

$$
\Delta \rho_{m}^{+}=\rho_{0} \cdot p_{1} \cdot \frac{M}{T} \cdot \frac{(1-\varepsilon)}{\varepsilon} \cdot\left\{1-(1-\varepsilon)^{m}\right\}
$$

Eq. (20) represents a monotonically increasing function of $m$, which has an asymptotic behaviour given by:

$$
\Delta \rho_{a}^{+}=\rho_{0} \cdot p_{1} \cdot \frac{M}{T} \cdot \frac{(1-\varepsilon)}{\varepsilon}
$$

Averaged demand failure probability over the period between maintenance activities $m$ $\underline{l \text { and } m, \rho_{m}}$

An averaged demand failure probability over the period between two consecutive maintenance activities $m-1$ and $m$ can be formulated using the following expression:

$$
\rho_{m}=\frac{1}{t_{m}-t_{m-1}} \int_{t_{m-1}}^{t_{m}} \rho_{m}(t) d t
$$

Substituting Eqs. (17) and (16) into Eq. (22) and making use of Eq. (12), the average demand failure probability between two consecutive maintenance activities $m-1$ and $m$ is given by:

$$
\rho_{m}=\rho_{0}+\rho_{0} \cdot p_{1} \cdot \frac{M}{T} \cdot\left\{\frac{1}{2}+\frac{(1-\varepsilon)}{\varepsilon} \cdot\left\{1-(1-\varepsilon)^{m-1}\right\}\right\}
$$

\section{Averaged demand failure probability over the renewal period $L, \rho^{*}$}

When a component is found to be in a serious degradation or failure condition, it will be replaced or effectively restored to an as-good-as-new (GAN) condition. Eq. (24) shows 
how the component renewal or overhaul maintenance is taken into account in the component demand-caused degradation model $[1,16]$.

$$
\rho^{*}=\frac{1}{m^{*}} \int_{0}^{m^{*}} \rho_{m} \mathrm{dm}
$$

Where $m^{*}=(L-M) / M$ and $L$ represents the renewal period. In actual practice, $L$ may vary. In this case, the $L$ can be taken as the average value [17].

Substituting Eq. (23) into Eq. (24) yields the following formulation of the average demand failure probability over the renewal period:

$$
\begin{aligned}
& \rho^{*}=\rho_{0}+\rho_{0} \cdot p_{1} \cdot \frac{M}{T} \cdot\left\{\frac{1}{2}+\frac{1}{m^{*}} \frac{(1-\varepsilon)}{\varepsilon} \int_{0}^{m^{*}}\left\{1-(1-\varepsilon)^{m-1}\right\} \mathrm{dm}\right\}= \\
& =\rho_{0}+\rho_{0} \cdot p_{1} \cdot \frac{M}{T} \cdot\left\{\frac{1}{2}+\frac{1}{m^{*}} \frac{(1-\varepsilon)}{\varepsilon} \cdot\left[\frac{(1-\varepsilon)^{m-1}}{\ln (1-\varepsilon)}\right]_{0}^{m^{*}}\right\} \\
& =\rho_{0}+\rho_{0} \cdot p_{1} \cdot \frac{M}{T} \cdot\left\{\frac{1}{2}+\frac{(1-\varepsilon)}{\varepsilon}-\frac{(1-\varepsilon)^{m^{*}}-1}{\varepsilon m^{*} \ln (1-\varepsilon)}\right\}
\end{aligned}
$$

Eq. (25) can be reformulated as follows:

$$
\rho^{*}=\rho_{0}+\Delta \rho^{*}
$$

Where $\Delta \rho^{*}$ represents the average increase of the component demand failure probability due to demand-caused degradation over the renewal period, which is given by:

$$
\Delta \rho^{*}=\rho_{0} \cdot p_{1} \cdot \frac{M}{T} \cdot\left\{\frac{1}{2}+\frac{(1-\varepsilon)}{\varepsilon}-\frac{(1-\varepsilon)^{m^{*}}-1}{\varepsilon m^{*} \ln (1-\varepsilon)}\right\}
$$

Eq. (27) represents a function of $m$, which has an asymptotic behaviour given by:

$$
\Delta \rho_{a}^{*}=\frac{1}{2} \rho_{0} \cdot p_{1} \cdot \frac{\mathrm{M}}{T} \cdot\left(\frac{2-\varepsilon}{\varepsilon}\right)
$$

\subsubsection{PAR model}


In the PAR approach, each maintenance activity is assumed to proportionally reduce, by a factor $\varepsilon$, only the component degradation gained from the previous maintenance, while the rest remains unaffected, where $\varepsilon$ represents the maintenance effectiveness that ranges in the interval $[0,1]$.

Time-dependent demand failure probability, $\rho_{m}(t)$

Similarly to the procedure followed for the PAS model, the value of the degradation function, $f($.$) , can be formulated considering the PAR approach. The degradation$ function immediately before and after the first maintenance activity is given by Eqs. (6) and (7), respectively. Next, $f($.$) for the time immediately before and after performing$ the second maintenance activity, named $f_{2}^{-}$and $f_{2}^{+}$respectively, can be formulated as follows:

$$
\begin{aligned}
& f_{2}^{-}(t)=p_{1} \cdot(1-\varepsilon)\left\lceil\frac{t_{1}}{T}\right\rceil+p_{1} \cdot\left[\frac{\left(t_{2}-t_{1}\right)}{T}\right\rceil \\
& f_{2}^{+}(t)=p_{1} \cdot(1-\varepsilon)\left\lceil\frac{t_{1}}{T}\right\rceil+p_{1} \cdot(1-\varepsilon)\left[\frac{\left(t_{2}-t_{1}\right)}{T}\right\rceil
\end{aligned}
$$

For the general case, the degradation function immediately after maintenance number $m$ is given by:

$$
f_{m}^{+}=p_{1} \cdot(1-\varepsilon) \cdot \frac{t_{m}}{T}
$$

Assuming preventive maintenance activities are performed on a regular basis with a constant maintenance interval given by $M$, Eq. (31) yields a monotonically increasing function of $m$ given by:

$$
f_{m}^{+}=p_{1} \cdot(1-\varepsilon) \cdot \frac{M}{T} \cdot m
$$

and similarly,

$$
f_{m}^{-}=p_{1} \cdot \frac{M}{T} \cdot((1-\varepsilon) \cdot(m-1)+1)
$$


Analogously to the process developed for PAS modelling, Eq. (17) can be particularized for $t=t_{m}$ immediately after maintenance number $m$, making use of Eqs. (16) and (32) now under the PAR model of the degradation function, which yields the formulation of the time-dependent demand failure probability immediately after maintenance $m$ :

$$
\rho_{\mathrm{m}}^{+}=\rho_{m}\left(t=t_{m}\right)=\rho_{0}+\rho_{0} \cdot p_{1} \cdot \frac{M}{T} \cdot(1-\varepsilon) \cdot m
$$

Eq. (34) can be reformulated as follows:

$$
\rho_{\mathrm{m}}^{+}=\rho_{0}+\Delta \rho_{m}^{+}
$$

Where $\Delta \rho_{m}^{+}$represents the increase in the time-dependent demand failure probability of the component due to accumulated demand-caused degradation up to having performed maintenance activity number $m$, which is given for the PAR model by:

$$
\Delta \rho_{m}^{+}=\rho_{0} \cdot p_{1} \cdot \frac{M}{T} \cdot(1-\varepsilon) \cdot m
$$

Average demand failure probability over the period between maintenance activities $m-1$ and $m, \rho_{m}$

The average demand failure probability over the period between two consecutive maintenance activities $m-1$ and $m$ can be formulated by Eq. (24), which is solved using Eq. (16) and (17) now making use of (32) under the PAR model, which yields:

$$
\rho_{m}=\rho_{0}+\rho_{0} \cdot p_{1} \cdot \frac{M}{T} \cdot\left\{\frac{1}{2}+(1-\varepsilon) \cdot(m-1)\right\}
$$

Average demand failure probability over the renewal period $L, \rho^{*}$

The average demand failure probability over the renewal period (useful life component $L$ ) can be formulated by Eqs. (26) and (37) to yield:

$$
\rho^{*}=\rho_{0}+\frac{1}{2} \cdot \rho_{0} \cdot p_{1} \cdot \frac{M}{T} \cdot\left\{1+(1-\varepsilon) \cdot\left(m^{*}-2\right)\right\}
$$


Where $m^{*}=(L-M) / M$ and $L$ represents the renewal period. Eq. (38) can be reformulated as follows:

$$
\rho^{*}=\rho_{0}+\Delta \rho^{*}
$$

Where $\Delta \rho^{*}$ represents the average increase of the component demand failure probability due to demand-caused degradation over the renewal period, which is given by:

$$
\Delta \rho^{*}=\frac{1}{2} \cdot \rho_{0} \cdot p_{1} \cdot \frac{M}{T} \cdot\left\{1+(1-\varepsilon) \cdot\left(m^{*}-2\right)\right\}
$$

\subsection{Addressing test efficiency}

The main objective of the surveillance test is to detect hidden failures so that the component can be restored to its operational state, normally the bad-as-old (BAO) state by performing corrective maintenance (CM) after the test has detected a failure. The effectiveness of the surveillance tests to detect a failure can be measured as proposed in Ref. [4], with a single parameter named test efficiency $(\eta)$.

As a result, the consideration of a test efficiency splits the total demand failure probability into two contributions: detected and undetected.

$\rho^{*}=\eta \cdot \rho^{*}+(1-\eta) \cdot \rho^{*}=\rho_{D}^{*}+\rho_{U}^{*}$

where the test efficiency, $\eta$, ranges in the interval $[0,1]$. In Eq. (41), the first contribution represents the demand failure probability associated with detected failures by testing, $\rho_{D}^{*}$, and the second part represents the demand failure probability associated with undetected failures by testing, $\rho_{U}^{*}$, which can be derived using the corresponding formulation for $\rho^{*}$ as introduced in Section 3.2, depending on whether the particular imperfect maintenance model adopted is the PAS or PAR model. 


\section{UNAVAILABILITY MODELLING}

The unavailability contributions of a component normally on standby are divided into two categories: a) unavailability due to failures, i.e. unreliability effect, and b) unavailability due to testing and maintenance downtimes, known as the downtime effect.

\subsection{Unreliability contribution}

The unreliability contribution to the unavailability of a component normally on standby has several terms, as introduced in Eq. (1). The only one of interest here is the demandcaused unreliability contribution, since only demand caused failure is considered in this paper.

Based on Eqs. (1) and (2), the demand-caused unreliability contribution is a function of the demand failure probability of the component in the period $m$, i.e. between maintenance $m-1$ and $m$, the time-dependent evolution of the unreliability contribution can be represented by:

$$
u_{R, m}(t)=\rho_{m}(t)
$$

Based on Eq. (24), it is simple to formulate an average unreliability contribution over the period between maintenance $m-1$ and $m$ as follows:

$$
u_{R, m}=\rho_{\mathrm{m}}
$$

Where $\rho_{\mathrm{m}}$ is the average demand failure probability of the component between two consecutive maintenance activities $m-1$ and $m$, which is given by Eqs. (23) or (37) under the assumption that the behaviour of the maintenance of the component follows a PAS or PAR model, respectively.

Based on Eq. (24), it is easy to formulate an average unreliability contribution over the renewal period of a component as follows: 


$$
u_{R}=\rho^{*}
$$

Where $\rho^{*}$ is the component average demand failure probability over the renewal period, which can be evaluated by Eqs. (25) or (38) under the assumption that the behaviour of the maintenance of the component follows a PAS or a PAR model, respectively. Under the PAS model, Eqs. (43) and (44) present an asymptotic behaviour for the largest $m$, as shown in Section 2.2.1.

\subsubsection{Unreliability doubling time consideration}

As can be seen in Eq. (42), the unreliability of a safety component normally on standby, which is both periodically tested and maintained, will increase over time as more tests are performed, even if further maintenance activities are performed.

The global increase in the component unreliability contribution can be represented by defining a component unreliability doubling time as proposed in Ref. [1]. In general, the doubling time may be understood as the chronological time at which the time-dependent unreliability contribution given by Eq. (42) reaches $u_{R, m}(t)=2 * \rho_{0}$. This general definition is adapted in this paper in accordance with Ref. [1], to take into account for the effect of maintenance described as follows.

Let us consider the time-dependent evolution of the component unreliability contribution given by Eq. (42), which represents a monotonically increasing function of $m$, which can be particularized for the PAS and PAR model for the time immediately after performing maintenance activity number $m$, i.e. $t=t_{m}$, using Eqns. (18) and (34) respectively to yield:

$$
u_{R, m}\left(t=t_{m}\right)=\rho_{m}^{+}=\left\{\begin{array}{cc}
\rho_{0}+\rho_{0} \cdot p_{1} \cdot \frac{M}{T} \cdot \frac{(1-\varepsilon)}{\varepsilon} \cdot\left\{1-(1-\varepsilon)^{m}\right\} & \text { PAS model } \\
\rho_{0}+\rho_{0} \cdot p_{1} \cdot \frac{M}{T} \cdot(1-\varepsilon) \cdot m & \text { PAR model }
\end{array}\right.
$$


Maintenance effectiveness reduces the unreliability contribution after each maintenance activity is performed. The doubling time is defined herein as the time, identified as $t_{D}$, at which maintenance activity number $m_{D}$ is performed, after which the component unreliability contribution is reduced but still remains above the doubling time condition $2 * \rho_{0}$. Therefore, this condition is reached by Eq. (45) when $u_{R, m}\left(t=t_{D}\right) \geq 2 * \rho_{0}$. This means that the second term of the sum on the right hand side of Eq. (45) must be set greater than or equal to the first term, i.e. $\rho_{0}$, to yield the maintenance activity number at which the doubling time is verified for each PAS and PAR model respectively:

$$
\mathrm{m}_{D} \geq \begin{cases}\frac{\ln \left\{1-\frac{T \varepsilon}{p_{1} M(1-\varepsilon)}\right\}}{\ln \{1-\varepsilon\}} & \text { PAS model } \\ \frac{T}{M \cdot p_{1}(1-\varepsilon)} & \text { PAR model }\end{cases}
$$

The component unreliability doubling time can then be estimated using the following relationship, $t_{D}=m_{D} \cdot M$ and Eq. (46). Thus, $m_{D}$ represents the maintenance activity after which the component unreliability will remain above the initial component unreliability. One could think about replacing the component at that time, i.e. performing a component renewal or overhaul maintenance, being $L=m_{D} \cdot M$.

\subsubsection{Average unreliability contribution over the renewal period}

The average unreliability contribution over the renewal period of a component is given by Eq. (44), which can be particularized for the PAS and PAR models, respectively:

$$
u_{R}=\left\{\begin{array}{cc}
\rho_{0}+\rho_{0} \cdot p_{1} \cdot \frac{M}{T} \cdot\left\{\frac{1}{2}+\frac{(1-\varepsilon)}{\varepsilon}-\frac{(1-\varepsilon)^{m^{*}}-1}{\varepsilon m^{*} \ln (1-\varepsilon)}\right\} & \text { PAS model } \\
\rho_{0}+\frac{1}{2} \cdot \rho_{0} \cdot p_{1} \cdot \frac{M}{T} \cdot\left\{1+(1-\varepsilon) \cdot\left(m^{*}-2\right)\right\} & \text { PAR model }
\end{array}\right.
$$

Where $m^{*}=(L-M) / M$ and $L$ represents the renewal period. 


\subsection{Downtime contributions}

In accordance with Ref. [4], the unavailability contributions due to detected downtimes for performing testing and maintenance activities with the plant at power, named the downtime effect, can be evaluated using the following equations:

$$
\begin{aligned}
& u_{T}=\frac{\tau}{T} \\
& u_{M}=\frac{\sigma}{M} \\
& u_{C}=\frac{1}{T} \rho_{D}^{*} \mu \\
& u_{O}=\frac{\theta}{L}
\end{aligned}
$$

where $u_{T}$ represents the unavailability contribution due to testing, $u_{M}$ is the unavailability contribution due to performing preventive maintenance, $u_{C}$ is the unavailability contribution due to performing/corrective maintenance conditional to detecting a failure during a previous test, and $u_{O}$ is the contribution due to replacement of the equipment, if any. In addition, the following new notation is used:

$\tau=$ downtime for testing,

$\sigma=$ downtime for preventive maintenance,

$\mu=$ downtime for corrective maintenance or repair, and

$\theta=$ downtime for replacement or renewal.

Then, the averaged unavailability of the component can be formulated by adding the several contributions introduced previously to yield:

$$
u=u_{R}+u_{T}+u_{M}+u_{C}+u_{O}
$$




\section{CASE STUDY}

This section describes the application of the proposed models to a typical motoroperated valve (MOV) in a nuclear power plant. Table 1 shows the reliability, ageing, testing and maintenance data of a $\operatorname{MOV}[15,16]$.

Table 1. Parameters for the base case

\begin{tabular}{ccc}
\hline Equipment & Value & [Units] \\
\hline$\rho_{0}$ & $1.82 \mathrm{E}-03$ & $1 /$ hour \\
\hline$p_{1}$ & $4.57 \mathrm{E}-10$ & $1 /$ demand \\
\hline $\mathrm{T}$ & 2190 & hour \\
\hline $\mathrm{M}$ & 13140 & hour \\
\hline$\varepsilon$ & 0.6 & \\
\hline$\eta$ & 0.6 & hour \\
\hline$\tau$ & 1 & hour \\
\hline$\sigma$ & 1 & hour \\
\hline$\mu$ & 2.6 & hour \\
\hline$\theta$ & 6 & hour \\
\hline $\mathrm{L}$ & 87600 &
\end{tabular}

\subsection{Time-dependent unreliability. PAS vs PAR models}

Firstly, the PAR and PAS models are compared to show the behaviour of the timedependent unreliability of the component under the PAS and PAR models. Fig. 1 shows the evolution of the demand failure probability with chronological time for both models. In this figure, it can be seen that the PAS model has asymptotic behaviour while the PAR model has a monotonically increasing behaviour. Due to this, the demand failure probability reaches the doubling time condition described in Section 3.1.1 (green dotted line) only for the PAR model. The black dotted line represents the doubling time, $t_{D}$, which is associated with the maintenance activity $m_{D}$ at which the component should be replaced, according to this doubling time condition. 
As regards the PAS model, the demand failure probability function of the component evolves over time but shows asymptotic behaviour. It is therefore possible to assume that constant maintenance keeps the age of the component at a constant or residual level in the long term, and that this depends on the maintenance effectiveness and on the test and maintenance intervals.

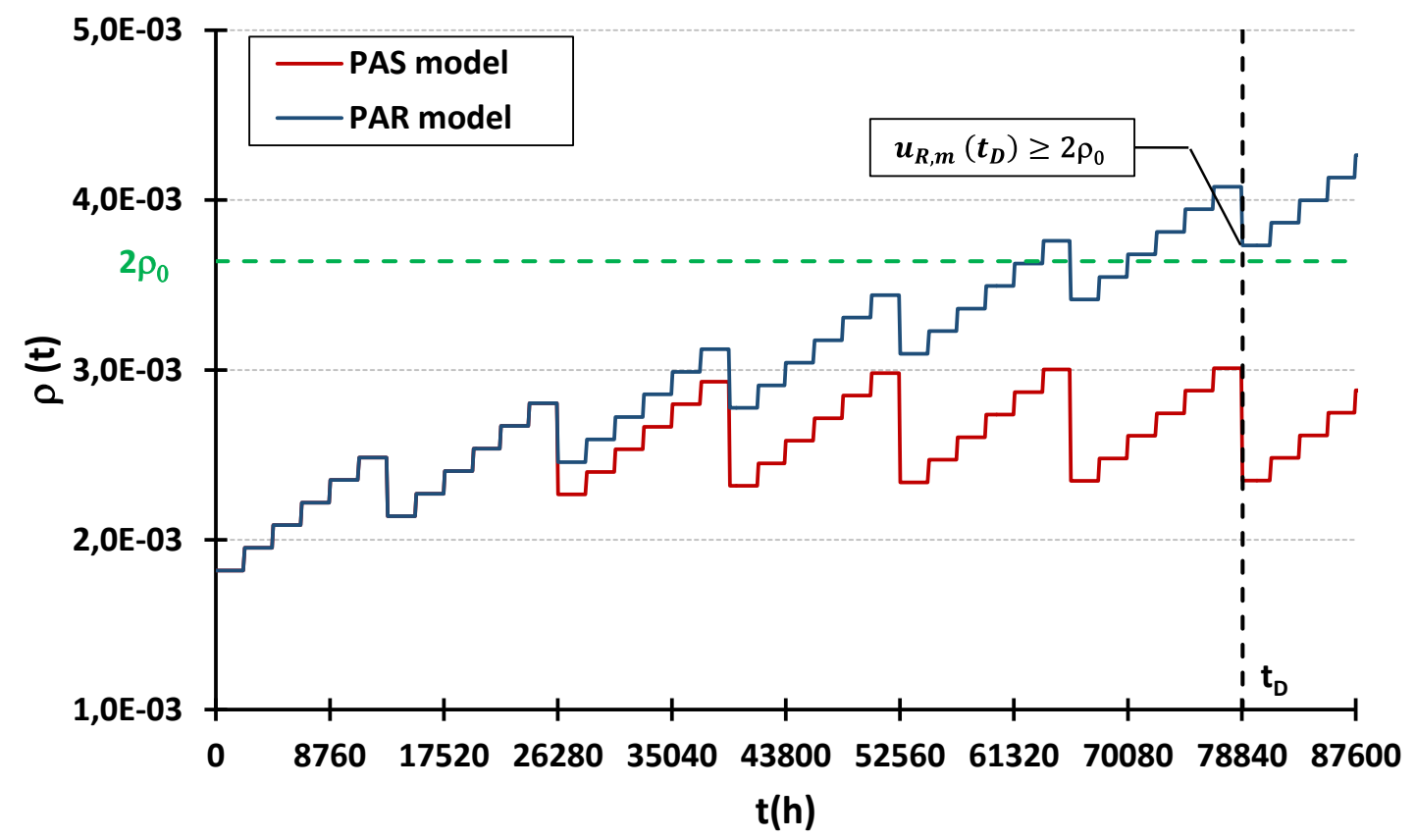

Fig. 1. $\rho(t)$ of the component under the PAS and PAR ageing models

\subsubsection{Sensitivity of $\rho(t)$ to maintenance interval}

Figs. 2 and 3 show the results of sensitivity analyses on $\rho(t)$ for different maintenance periods $M$ under PAS and PAR models, respectively. In the PAS model case it can be seen in Fig. 2 that, while keeping constant maintenance effectiveness $\varepsilon=0.6$, if the period is extended the asymptotic behaviour is reached later and $\rho(t)$ becomes higher. Thus, for $M=18$ months, the asymptotic value $\rho_{\mathrm{m}}^{+}$tends to $2.35 \mathrm{E}-03$, which is reached after $52560 \mathrm{~h}$. This asymptotic behaviour is not observed in the rest of the cases, as they occur later. The demand failure probability reaches twice its initial earlier value for $M=3$ years, i.e. the doubling time for this maintenance interval is approximately 45000 $\mathrm{h}$, while the doubling time is approximately $30000 \mathrm{~h}$ for $M=5$ years. On the other hand, under the PAR model, see Fig. 3, doubling times are approximately 28000 h, $45000 \mathrm{~h}$ and $60000 \mathrm{~h}$ for $M=18$ months, $M=3$ years and $M=5$ years, respectively. 


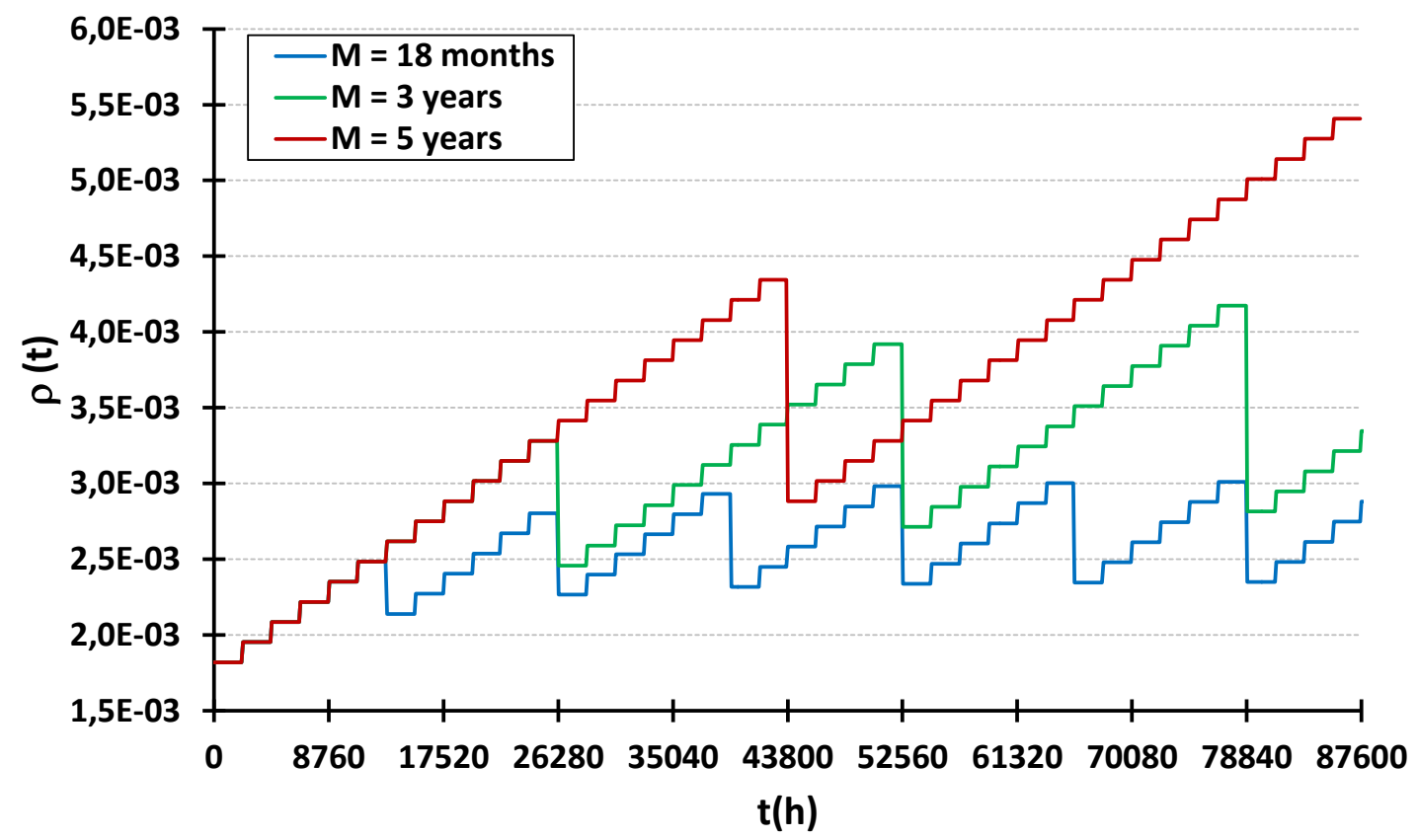

Fig. 2. $\rho(t)$ of the component for different maintenance periods under PAS model

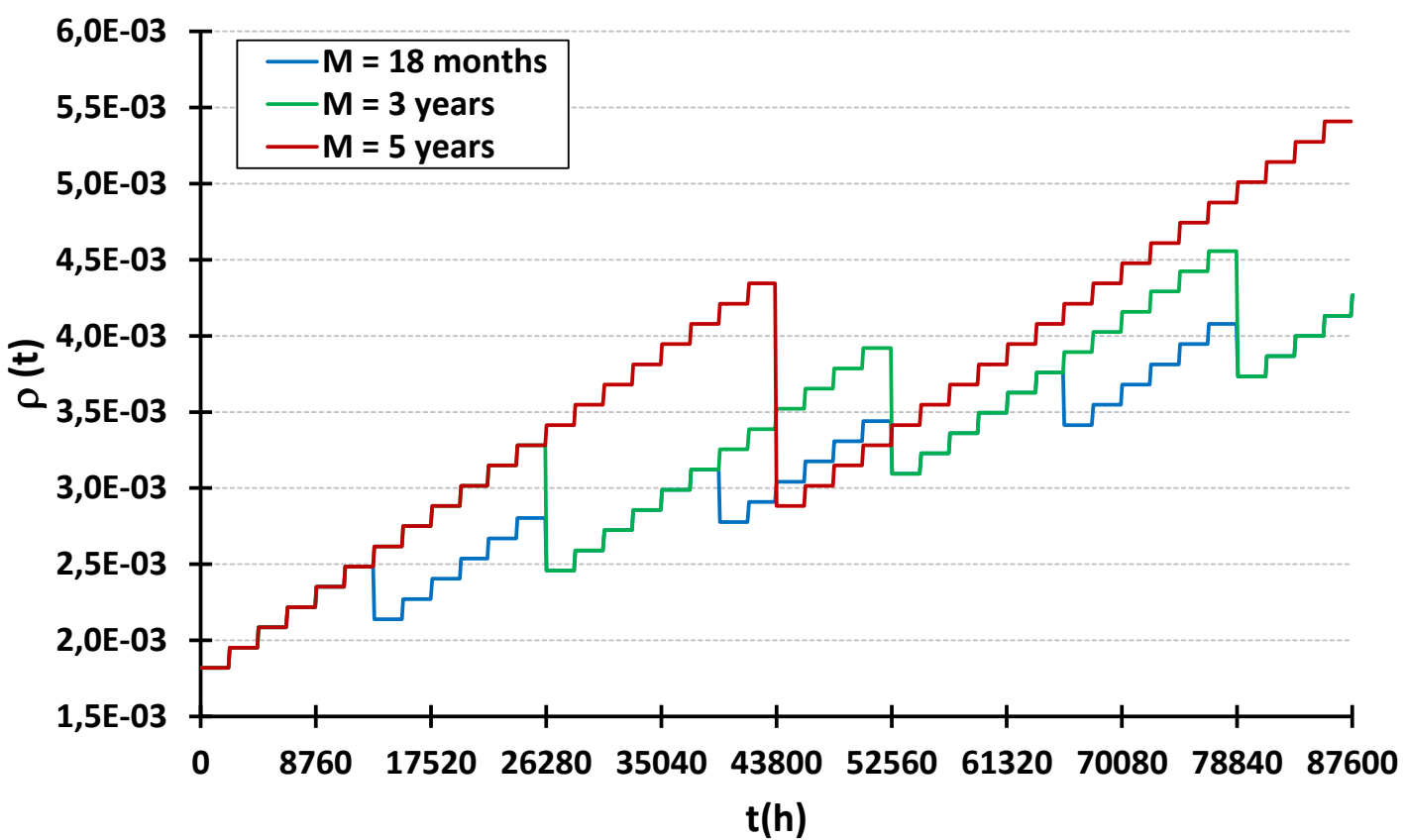

Fig. 3. $\rho(t)$ of the component for different maintenance periodsunder PAR model 


\subsubsection{Sensitivity of $\rho(t)$ to maintenance effectiveness}

Figs. 4 and 5 show the results of the sensitivity analyses on the $\rho(t)$ for different maintenance effectiveness values, $\varepsilon$, under the PAS and PAR models, respectively. The maintenance interval is kept constant at $M=18$ months. For the PAS model, as maintenance effectiveness increases, the asymptotic behaviour is reached earlier. This is a natural consequence of having a maintenance activity that is able to further improve the state of the component. Thus, while for $\varepsilon=0.4$ the asymptotic value is not achieved after 10 years, the asymptotic value for $\varepsilon=0.6$ tends to $2.35 \mathrm{E}-03$ and for $\varepsilon=0.8$ the asymptotic value tends to $1.99 \mathrm{E}-03$. The doubling time is not achieved in any of the cases studied, however, the evolution almost reaches this doubling time for the worst maintenance effectiveness, i.e. $\varepsilon=0.4$. On the other hand, as expected, the doubling time is reached for the PAR model, and since the slope of the evolution of the demand failure probability increases when maintenance effectiveness decreases, the doubling time is reached sooner.

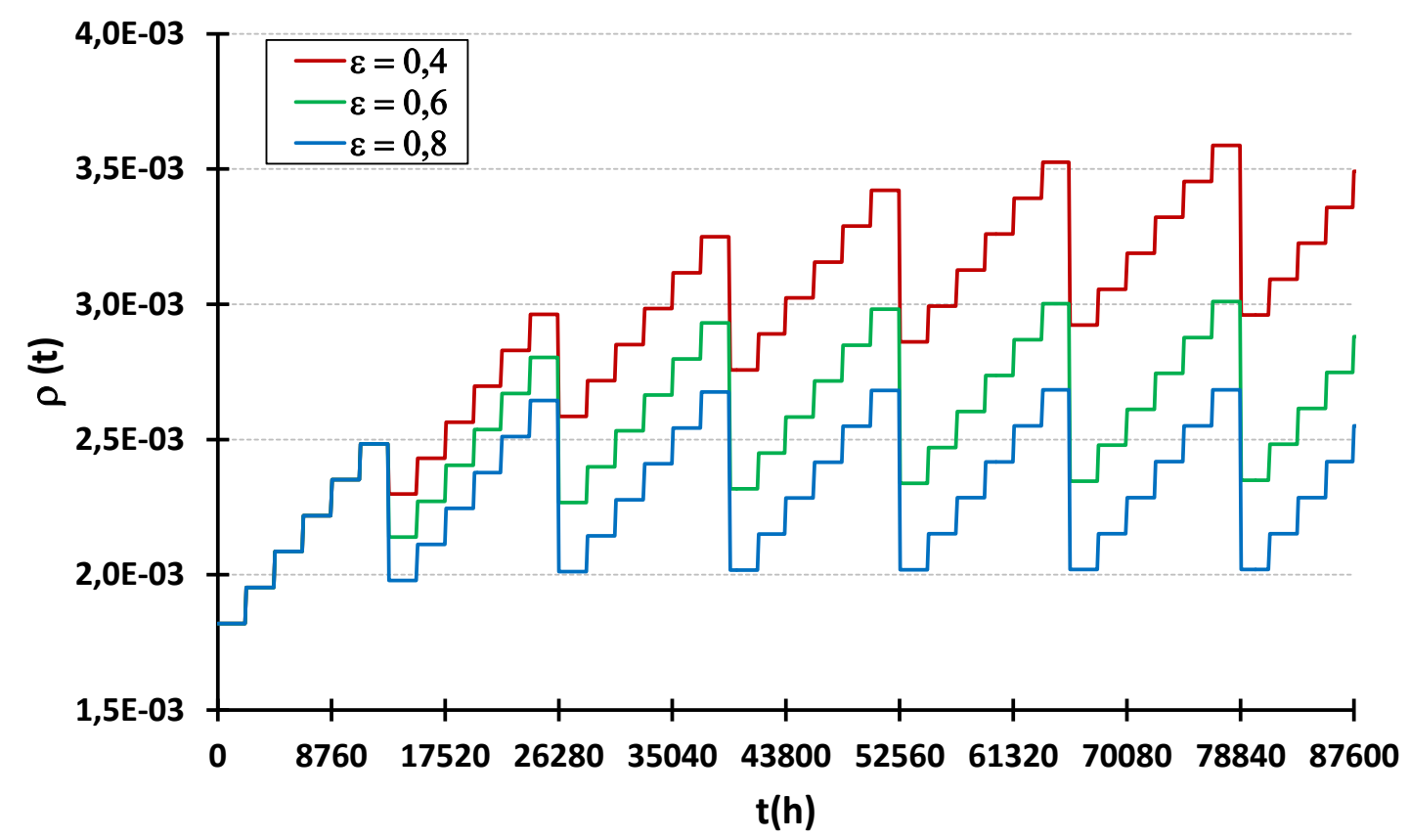

Fig. 4. $\rho(t)$ of the component for different maintenance effectiveness under PAS model. 


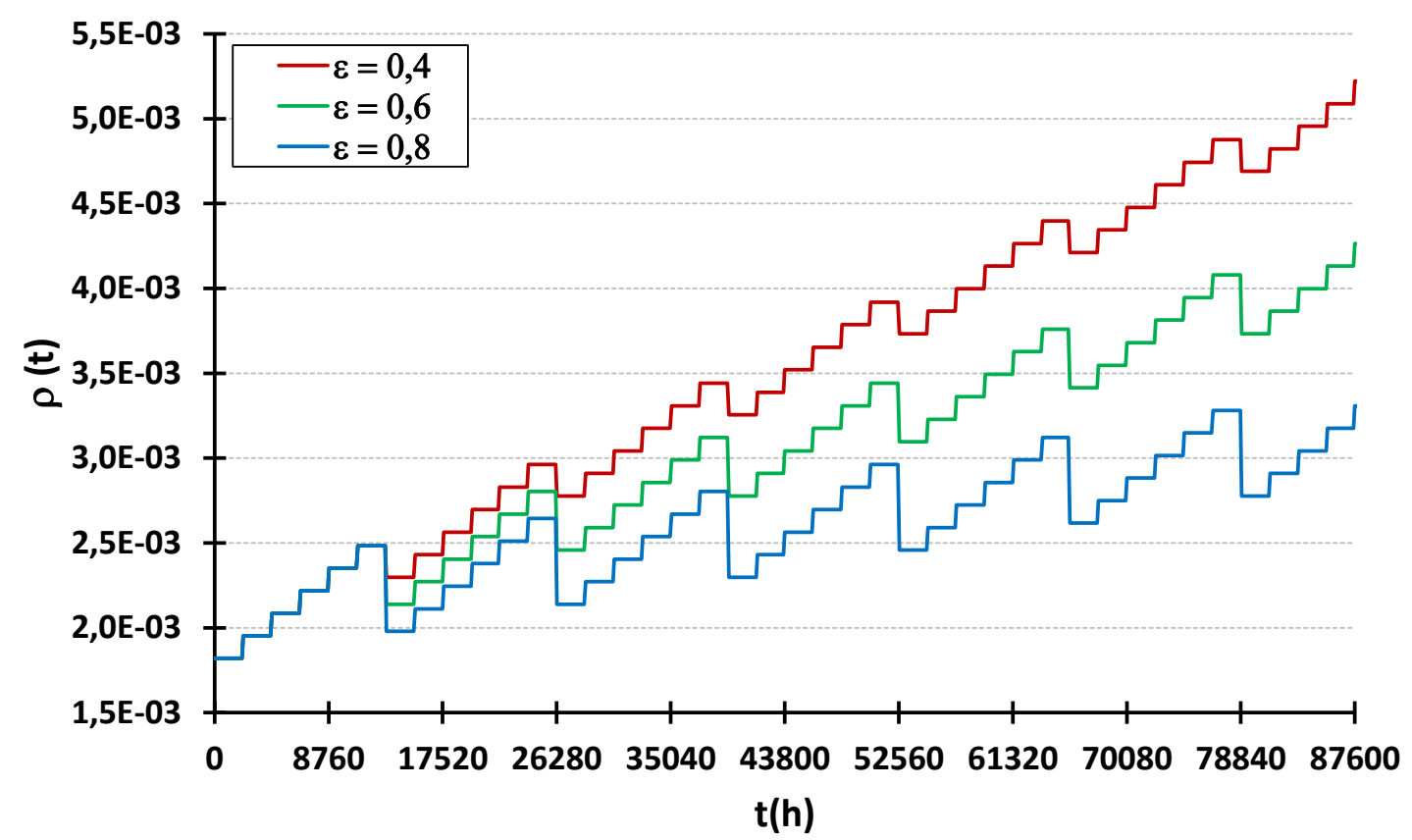

Fig. 5. $\rho(t)$ of the component for different maintenance effectiveness under PAR model.

\subsection{Unreliability doubling time sensitivity to maintenance and test intervals}

The next sensitivity study involyes the analysis of the influence of maintenance and test related parameters on doubling time, $t_{D}$, given by Eq. (46).

The unreliability doubling time is not reached for the evolution of the demand failure probability under the PAS model for the base case using the data in Table 1 (see Fig. 1). However, it is shown that such doubling time may be reached even under the PAS model, depending on the value of the maintenance related parameters (see Figs. 2 and 4). Fig. 6 shows possible combinations of the $M / T$, test degradation factor and maintenance effectiveness values for which doubling time is reached. 


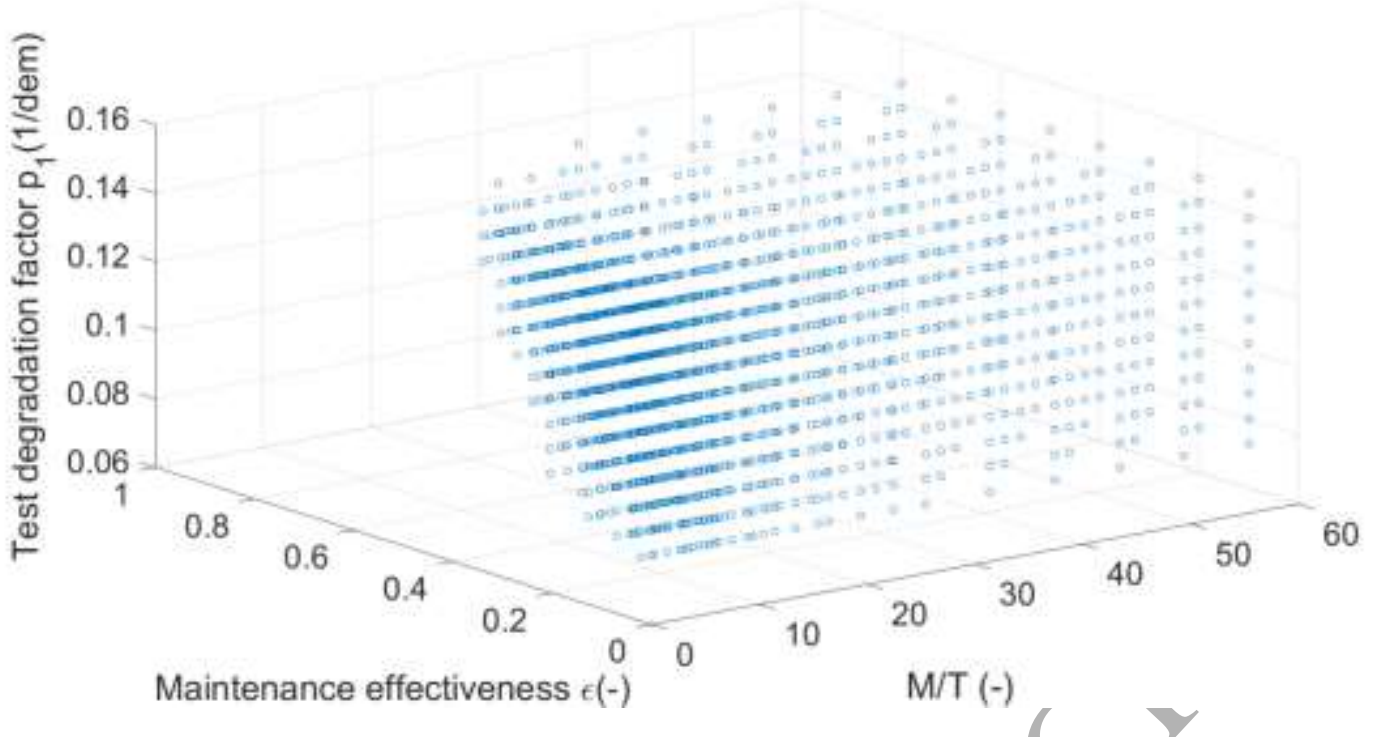

Fig. 6. Possible combinations of $p_{1}, \varepsilon$ and $M / T$ under PAS model to reach the doubling time before 10 years.

On the other hand, the unreliability doubling time is reached for the evolution of the demand failure probability under the PAR model for the base case using the data in Table 1 (see Fig. 1). Fig.7 shows how the doubling time depends on $M$ and $T$, fixing $p_{1}$ and $\varepsilon$ at their initial values in Table 1. An inversely proportional dependence between $t_{D}$ and $T$ is clearly observed, as can be seen by analysing Eq. (46) for the PAR model, which shows that $t_{D}$ does not depend on $M$.

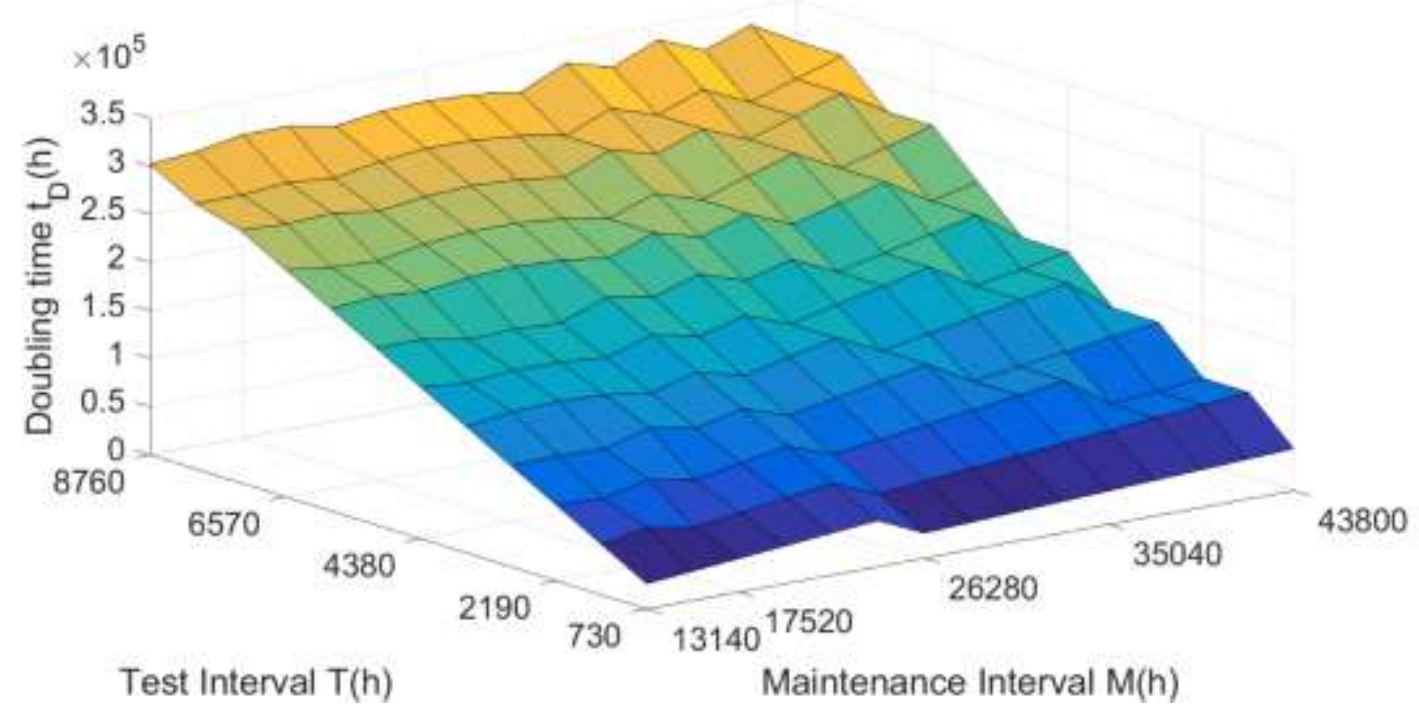

Fig. 7. $t_{D}$ for different maintenance and test intervals under PAR model. 


\subsection{Average unavailability as a function of maintenance and test intervals}

The last sensitivity study involves the evolution of the total unavailability of the component, given by Eq. (52), as a function of the couple $\{M, T\}$, i.e. maintenance and test intervals, for constant $p_{1}, \varepsilon$ and $\eta$ parameters, as shown in Table 1. In particular, the test efficiency, $\eta$, had no impact on $\rho(t)$ but it affects component unavailability, $u$, as a function of $u_{C}$, see Eq. (50).

Fig. 8 shows the results achieved under the PAS model. The highest values of $u$ are reached adopting a high maintenance interval and low test interval The main contributor to the total unavailability, $u$, (see Eq. (52)), is the unreliability effect given by Eq. (47). This explains the observed inversely proportional dependence between $u$ and $T$, as one can realize from Eq. (47) under the PAS model, while there is an almost proportional dependence between $u$ and $M$.
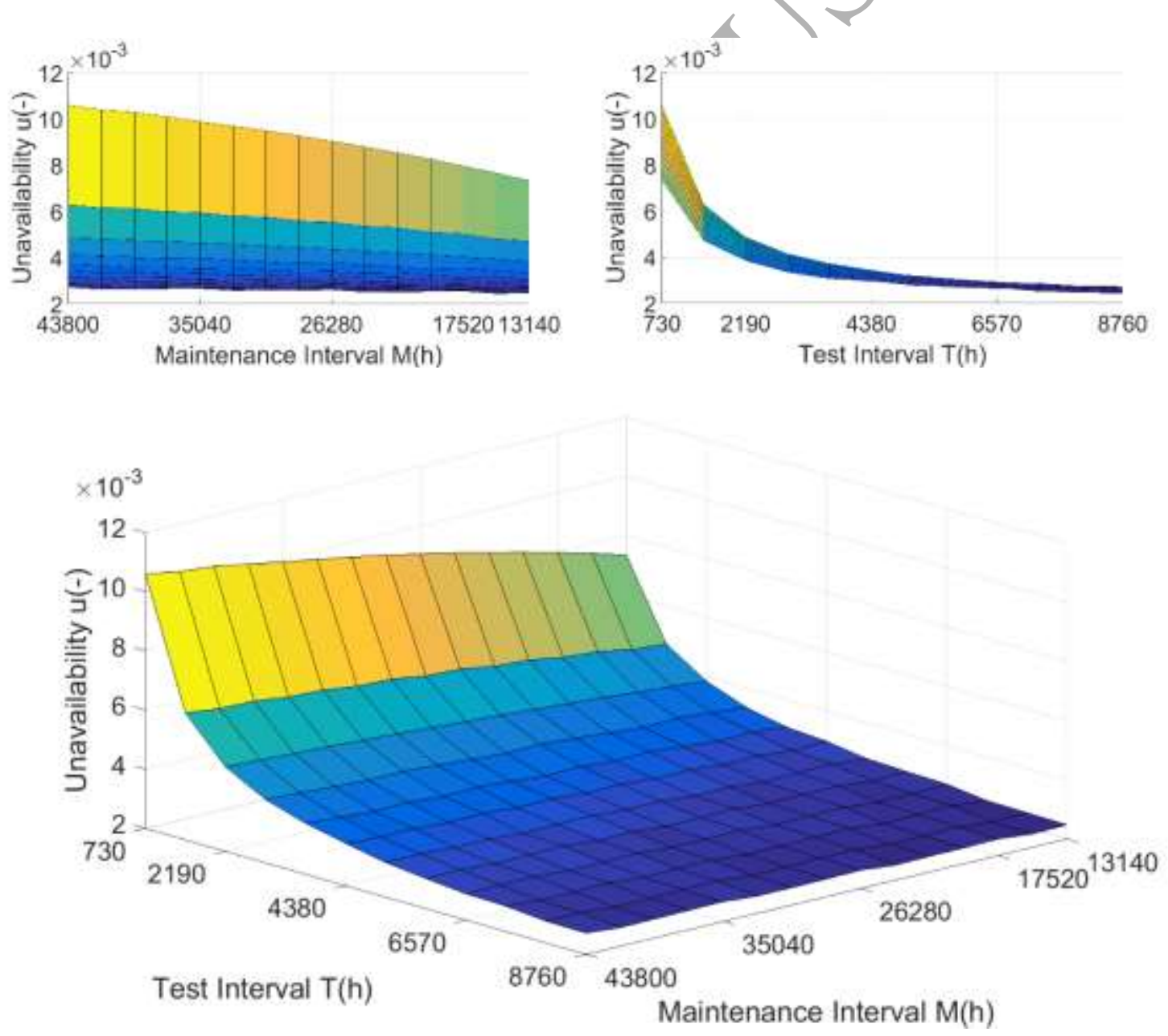
Fig. 8. Unavailability for different maintenance and test intervals under PAS model.

Fig. 9 shows the results achieved under the PAR model. Again, the highest values of $u$ are reached adopting a high maintenance interval and low test interval. The main contributor to total unavailability is the component unreliability given by Eq. (47), which explains the inversely proportional dependence between $u$ and $T$ observed once more, as can be seen in Eq. (47) under the PAR model, while there is an almost proportional dependence between $u$ and $M$.
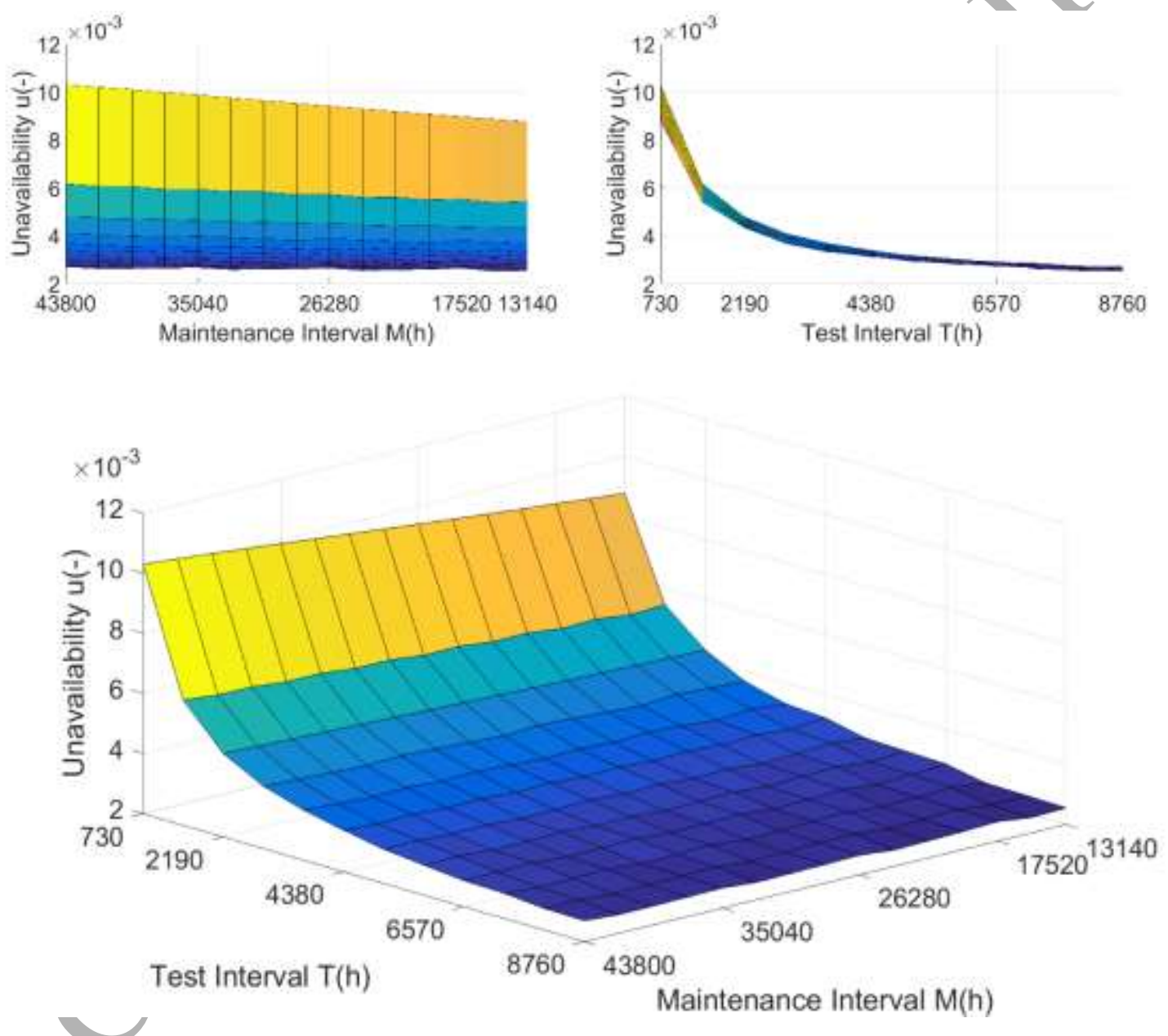

Fig. 9. Unavailability for different maintenance and test intervals under PAR model.

Comparing the unavailability results from Fig. 8 and 9, it can be seen that the total unavailability is higher for the PAR than the PAS model, as a consequence of the asymptotic behaviour of the PAS model. For this reason $u$ increases faster for the PAR model than for the PAS model with the same values of $M$ and $T$. See for example the 
value reached by $u$ for the couple of the lowest $T$ and the highest $M$ values as compared to the value reached by $u$ for the couple of the highest $T$ and lowest $M$ values.

\section{CONCLUDING REMARKS}

The reliability, availability and maintainability (RAM) modelling of safety equipment has long been a topic of major concern. Some RAM models have focussed on explicitly addressing the effect of component degradation and surveillance and maintenance policies.

These models are intended to be used as a tool to search for an optimum RAM level of safety components by adjusting the design, surveillance and maintenance related parameters, among other factors. Consequently, they must not only account for the positive but also the negative effects of surveillance and maintenance activities.

As regards the RAM reliability components, these normally present two main types of failure modes on demand: (1) by demand-caused and (2) standby-related failures. The former is normally associated with a demand failure probability while the other is associated with a standby failure rate. Both failure modes are often affected by degradation, such as demand-related stress and ageing, which cause the component to degrade and ultimately to fail.

Most previous studies have/concentrated on the effect of component ageing and paid less attention to demand-related stress. However, the demand failure probability is manly affected by the degradation caused by demand-related stress, so that surveillance testing introduces not only a positive but also an adverse effect, which is compensated by performing maintenance activities that are intended to eliminate or reduce the accumulated degradation.

This paper proposes a new model for the demand failure probability of a safety component that explicitly addresses all aspects of the effect of demand-induced stress (mainly test-induced stress), maintenance effectiveness (based on a PAS or PAR imperfect maintenance modelling) and test efficiency. 
To demonstrate the new model's performance, it was applied to a typical motoroperated valve in a nuclear power plant, which is normally influenced by test-caused degradation affecting its demand failure probability. The model was able to faithfully reproduce the influence of all the relevant maintenance and test related parameters.

The example of the application clearly shows a major qualitative difference between using PAS or PAR as the imperfect maintenance model, since the former shows asymptotic behaviour. This affects the existence of the unreliability doubling time and the evolution of the unreliability contribution to the component's unavailability. In addition, the sensitivity studies on the maintenance and test-related parameters show the impact of maintenance and test intervals, maintenance effectiveness and degradation factor on both the doubling time and the unreliability contribution under both the PAS and PAR models.

It can therefore be concluded that before using the proposed model it is important to appropriately calibrate the applicable imperfect maintenance model and to estimate the corresponding maintenance and test related parameters for each particular application case.

\section{ACKNOWLEDGMENTS}

The authors are grateful to the Spanish Ministry of Science and Innovation for the financial support received (Research Projects ENE2013-45540-R and ENE2016-80401R) and the doctoral scholarship awarded (BES-2014-067602). The study also received financial support from the Spanish Research Agency and the European Regional Development Fund.

\section{REFERENCES}

[1] Kim, I. S., Martorell, S., Vesely, W. E. \& Samanta, P. K., Quantitative evaluation of surveillance test intervals including test-caused risks. NUREG/CR-5775, BNL-NUREG-52296, Feb. 1992. 
[2] Kim IS, Martorell S a, Vesely WE, Samanta PK., Risk analysis of surveillance requirements including their adverse effects. Reliability Engineering and System Safety 1994; 45: 225-34.

[3] Martorell S, Sanchez A, Serradell V, Age-dependent reliability model considering effects of maintenance and working conditions. Reliability Engineering and System Safety 1999; 64: 19-31.

[4] Martón I, Sánchez AI, Martorell S, Ageing PSA incorporating effectiveness of maintenance and testing. Reliability Engineering and System Safety 2015; 139: 131-40.

[5] Volkanovski A., Method for assessment of ageing based on PSA results. Nuclear Engineering and Design2012, 246: 141-146.

[6] D. Kančev, M. Čepin, B. Gjorgiev, Development and application of a living probabilistic safety assessment tool: multi-objective multi-dimensional optimization of surveillance requirements in NPPs considering their ageing. Reliability Engineering and System Safety 2014, 131: 135-147.

[7] Jussi K. Vaurio, A recursive framework for time-dependent characteristics of tested and maintained standby units with arbitrary distributions for failures and repairs. Reliability Engineering and System Safety2015, 138: 154-162.

[8] I. Martón, ,P. Martorell, R. Mullor, A.I. Sánchez, S. Martorell, Optimization of test and maintenance of ageing components consisting of multiple items and addressing effectiveness. Reliability Engineering and System Safety2016, 153: 151-158.

[9] Li Yang, Xiaobing Ma, Yu Zhao, A condition-based maintenance model for a three-state system subject to degradation and environmental shocks. Computers \& Industrial Engineering 2017, 105: 210-222.

[10] Sun Yeong Choi, Jooho Lee, Joon-Eon Yang, An analysis for risk impact of Emergency Diesel Generator on modified Surveillance Test Interval by considering failure due to demand stress.Nuclear Engineering and Design2010, 240: 3577-3581.

[11] Jian Zhang, Xiaoyan Huang, Youtong Fang, Jing Zhou, He Zhang, Jing Li, Optimal inspection-based preventive maintenance policy for three-state mechanical components under competing failure modes.Reliability Engineering and System Safety 2016, 152: 95-103.

[11] N.C. Caballé, I.T. Castro, C.J. Pérez, J.M. Lanza-Gutiérrez, A condition-based maintenance of a dependent degradation-threshold-shock model in a system with multiple degradation processes. Reliability Engineering \& System Safety 2015, 34,: 98 109. 
[13] Torres-Echeverría AC, Martorell S, Thompson HA. Modelling and optimization of proof testing policies for safety instrumented systems. Reliability Engineering and System Safety 2009; 94: 838-54.

[14] Torres-Echeverría, A. C., Martorell, S., \& Thompson, H. A.,Modeling safety instrumented systems with MooN voting architectures addressing system reconfiguration for testing. Reliability Engineering and System Safety2011, 96(5): 545563.

[15] Sung Min Shin, In Seop Jeon, Hyun Gook Kang., Surveillance test and monitoring strategy for the availability improvement of standby equipment using agedependent model. Reliability Engineering and System Safety2015,135: 100-106.

[16] Sanchez A, Carlos S, Martorell S, Villanueva J.F., Addressing imperfect maintenance modelling uncertainty in unavailability and cost based optimization. Reliability Engineering and System Safety 2009, 94: 22-32.

[17] W.E. Vesely, R.E. Kurth and S.M Scalzo, Evaluation of core melt frequency effects due to component ageing and maintenance, NUREG/CR-5510, June 1990. 